parenting and shared attention). Assessments may identify at-risk families for early intervention, but further work is required to develop and validate reliable methods for risk stratification in community-based practice.

\section{Factors in psychiatric admissions: before and during the COVID-19 pandemic}

\author{
Robyn McCarron ${ }^{1 \star}$, Peter Swann ${ }^{2}$, Fiona Thompson ${ }^{1}$ \\ and Graham Murray ${ }^{2}$ \\ ${ }^{1}$ Cambridgeshire and Peterborough NHS foundation trust and \\ ${ }^{2}$ University of Cambridge, Cambridgeshire and Peterborough NHS \\ foundation trust \\ ${ }^{\star}$ Corresponding author.
}

doi: 10.1192/bjo.2021.156

Aims. The COVID-19 pandemic has impacted community mental health, but the effect on psychiatric admissions is unknown. We investigated factors contributing to acute psychiatric admissions, and whether this changed during the first UK lockdown.

Method. A retrospective case-note review study with an exploratory mixed-methods design was used to examine factors in psychiatric admissions following the first UK 2020 lockdown compared to the same time periods in 2019 and 2018.

Result. Themes of psychopathology, risk, social stressors, community treatment issues, and physical health concerns were generated. The mean number of codes per case was $6 \cdot 19$ (s.d. $=2 \cdot 43$ ), with a mean number of categories per case of $3 \cdot 73$, (s.d. $=0.98)$. Changes in routines and isolation were common factors in the study year; accommodation and substance abuse were more prominent in the control year. Relationship stressors featured strongly in both groups. There were significantly more women $(\chi 2(1, \mathrm{~N}=98)=20.80, \mathrm{p}<0.00001)$ and older adults $(\chi 2(1$, $\mathrm{N}=98)=8.61, \mathrm{p}=0.0033)$ in the study group than the control. Single people, compared to those in a relationship $(\chi 2(1, \mathrm{~N}=$ $45)=4.46, \mathrm{p}=0.035)$, and people with affective disorders compared to psychotic disorders $((\chi 2(1, \mathrm{~N}=28)=5.19, \mathrm{p}=0 \cdot 023)$, were more likely to have a COVID-19 related admission factor.

Conclusion. The COVID-19 pandemic amplified pre-existing psychosocial vulnerabilities with a disproportionate psychiatric admissions impact on the mental health of women, the elderly and those with affective disorders.

\section{Vive la difference! Celebrating and supporting autistic psychiatrists with autistic doctors international}

Sue McCowan ${ }^{1 \star}$, Sebastian C K Shaw ${ }^{2}$, Mary Doherty ${ }^{3}$, Bernadette Grosjean ${ }^{4}$, Paula Blank ${ }^{5}$ and Malcolm Kinnear ${ }^{6}$

${ }^{1}$ Associate Specialist Doctor, Old Age Psychiatry, Dorset Healthcare University NHS Foundation Trust; ${ }^{2}$ Honorary Clinical Lecturer, Department of Medical Education, Brighton and Sussex Medical School; ${ }^{3}$ Founder of Autistic Doctors International, Consultant Anaesthetist, Our Lady's Hospital, Navan; ${ }^{4}$ Retired Associate Professor of Psychiatry, University of California Los Angeles; ${ }^{5}$ Consultant Child and Adolescent Psychiatrist, UK and ${ }^{6}$ Consultant General Adult Psychiatrist, NHS Fife, Honorary Senior Clinical Teacher, University of Dundee

${ }^{*}$ Corresponding author.

doi: 10.1192/bjo.2021.157

Aims. We aim to raise awareness of the existence and value of autistic doctors in psychiatry and to also signpost psychiatrists who are or suspect they might be autistic towards peer support.

Method. Autism refers to a lifelong difference in how people communicate and interact with the world. These differences lead to strengths and challenges with individual profiles which include special interests, hyper-focus, and often sensory differences and anxiety. Autism has an estimated prevalence of $1-2 \%$, which is likely an underestimate. It was noted that there was little in the way of advocacy for autistic doctors around the world. Anecdotal evidence also suggested possible issues of misunderstanding and stigmatisation of autistic doctors. As such, there was a need to tackle this to promote positive change. MD founded the group Autistic Doctors International (ADI) in 2019 to foster camaraderie, advocacy and support. ADI has flourished with 250+ members currently. In a recent member poll, 24 of 180 respondents identified themselves as psychiatrists - second only to general practice $(n=54)$. Several other consultant psychiatrists are known to self-identify as autistic but have not formally joined due to the fear of disclosure. The group has additionally supported multiple doctors to tackle prejudice and discrimination in the workplace / training environment. It has also brought together autistic doctors with academic interests and has generated multiple academic outputs in the form of publications, research grants and conference posters/papers regarding autism. Result. Psychiatrists, and doctors in general, are a self-selecting group for many autistic strengths such as hyper-focus, curiosity, self-motivation, a desire to study social communication, attention to detail, pattern recognition, problem solving and empathy, which, contrary to prevailing stereotypes, can be marked in autism. The increasing numbers of doctors joining ADI supports the assumption that autistic individuals are safe and effective clinicians. It is worth noting that many members are not 'doctors in difficulty'. Those who have been able to achieve suitable accommodations, often without realising why they were needed, have flourished. Such accommodations and outcomes are in line with the neurodiversity movement, which promotes a view of autism as difference, rather than pure disability or disorder. This aims to challenge stereotypes and the tragedy narrative surrounding autism.

Conclusion. Autism awareness is increasing amongst doctors but more open discussion is still needed in order to facilitate appropriate peer and workplace support. This is likely to improve mental wellbeing and resilience for autistic psychiatrists.

Physical morbidity and mortality in male adolescent anorexia: a scoping review

\section{Craig McEwan}

Sussex Partnership NHS Foundation Trust (SPFT)

doi: $10.1192 /$ bjo.2021.158

Aims. Anorexia Nervosa (AN) mimics a state of starvation as a result of extreme calorific restriction, often with associated extreme exercise or purging behaviours. The physiological demands are known to lead to a number of health complications and contribute to a significantly increased mortality compared to the general population. Although males account for $10 \%$ of the AN population, they are often underrepresented in research. There is a particular gap in evidence for males under 18 despite the unique physiological requirements of adolescence including growth, puberty and achieving peak bone mass.

This review aims to bring together current research on physical health complications in male adolescent anorexia and help understand the knowledge gaps which exist. 